ANNALES

POLONICI MATHEMATICI

LXXVII.3 (2001)

\title{
Comparison theorems for infinite systems of parabolic functional-differential equations
}

\author{
by Danuta Jaruszewska-Walczak (Gdańsk)
}

\begin{abstract}
The paper deals with a weakly coupled system of functional-differential equations

$$
\partial_{t} u_{i}(t, x)=f_{i}\left(t, x, u(t, x), u, \partial_{x} u_{i}(t, x), \partial_{x x} u_{i}(t, x)\right), \quad i \in S,
$$

where $(t, x)=\left(t, x_{1}, \ldots, x_{n}\right) \in(0, a) \times G, u=\left\{u_{i}\right\}_{i \in S}$ and $S$ is an arbitrary set of indices. Initial boundary conditions are considered and the following questions are discussed: estimates of solutions, criteria of uniqueness, continuous dependence of solutions on given functions. The right hand sides of the equations satisfy nonlinear estimates of the Perron type with respect to the unknown functions. The results are based on a theorem on extremal solutions of an initial problem for infinite systems of ordinary functional-differential equations.
\end{abstract}

1. Introduction. For any metric spaces $X$ and $Y$, let $C(X, Y)$ denote the class of all continuous functions from $X$ to $Y$. Put $D=(0, a) \times G$ where $a>0$ and $G \subset \mathbb{R}^{n}$ is an open bounded domain. Let $D_{0}=\left[-r_{0}, 0\right] \times \bar{G}$ and $\Sigma=(0, a) \times \partial G$ where $r_{0} \geq 0$ and $\partial G$ is the boundary of $G$. Write $\Delta=D_{0} \cup D \cup \Sigma$.

Let $S$ be an arbitrary set of indices and

$$
X=\left\{p=\left\{p_{k}\right\}_{k \in S}: p_{k} \in \mathbb{R}, k \in S\right\} .
$$

For $p, \bar{p} \in X$ where $p=\left\{p_{k}\right\}_{k \in S}, \bar{p}=\left\{\bar{p}_{k}\right\}_{k \in S}$ we write $p \leq \bar{p}$ if $p_{k} \leq \bar{p}_{k}$ for $k \in S$.

For a sequence $\left\{p^{(m)}\right\}_{m \in \mathbb{N}}, p^{(m)}=\left\{p_{k}^{(m)}\right\}_{k \in S} \in X$, and for a point $p=$ $\left\{p_{k}\right\}_{k \in S} \in X$ we write $\lim _{m \rightarrow \infty} p^{(m)}=p$ if $\lim _{m \rightarrow \infty} p_{k}^{(m)}=p_{k}$ for all $k \in S$.

Denote by $|\cdot|_{0}$ the supremum norm in the space of bounded real continuous functions defined on $\Delta$. For a sequence $\left\{u^{(m)}\right\}_{m \in \mathbb{N}}, u^{(m)}=\left\{u_{k}^{(m)}\right\}_{k \in S} \in$ $C(\Delta, X)$, and for a function $u=\left\{u_{k}\right\}_{k \in S} \in C(\Delta, X)$, we write $\lim _{m \rightarrow \infty} u^{(m)}$ $=u$ if $\lim _{m \rightarrow \infty}\left|u_{k}^{(m)}-u_{k}\right|_{0}=0$ for all $k \in S$.

2000 Mathematics Subject Classification: 35R45, 35K50.

Key words and phrases: nonlinear estimates of the Perron type, infinite systems of nonlinear parabolic functional-differential equations. 
Let $M_{n \times n}$ denote the set of all real $n \times n$ matrices. For $r, \widetilde{r} \in M_{n \times n}$, $r=\left[r_{j k}\right]_{j, k=1, \ldots, n}, \widetilde{r}=\left[\widetilde{r}_{j k}\right]_{j, k=1, \ldots, n}$, we write $r \leq \widetilde{r}$ if

$$
\sum_{j, k=1}^{n}\left(r_{j k}-\widetilde{r}_{j k}\right) \lambda_{j} \lambda_{k} \leq 0 \quad \text { for any } \lambda=\left(\lambda_{1}, \ldots, \lambda_{n}\right) \in \mathbb{R}^{n} .
$$

Write $\Gamma=D \times X \times C(\Delta, X) \times \mathbb{R}^{n} \times M_{n \times n}$ and let $f=\left\{f_{i}\right\}_{i \in S}, f_{i}: \Gamma \rightarrow \mathbb{R}$, and $\alpha=\left\{\alpha_{i}\right\}_{i \in S}, \alpha_{i}: D_{0} \cup \Sigma \rightarrow \mathbb{R}$, be given functions.

Let a function $c=\left\{c_{i}\right\}_{i \in S}, c_{i}: \Sigma \rightarrow \mathbb{R}_{+}$, be given. Define

$$
\Sigma_{c_{i}}=\left\{(t, x) \in \Sigma: c_{i}(t, x) \neq 0\right\} .
$$

Assume that a function $b=\left\{b_{i}\right\}_{i \in S}, b_{i}: \Sigma_{c_{i}} \rightarrow \mathbb{R}_{+}$, is given and $b_{i}(t, x) \geq$ $B_{i}>0$ on $\Sigma_{c_{i}}$ for $i \in S$.

Suppose that for every $i \in S$ and $(t, x) \in \Sigma_{c_{i}}$ there is a direction $l_{i}(t, x)$ $\in \mathbb{R}^{n}$ such that for some $h_{0}>0$,

$$
\left(t, x+h l_{i}(t, x)\right) \in \bar{D}, \quad h \in\left[0, h_{0}\right] .
$$

We consider the initial boundary value problem

$$
\begin{aligned}
\partial_{t} u_{i}(t, x) & =f_{i}\left(t, x, u(t, x), u, \partial_{x} u_{i}(t, x), \partial_{x x} u_{i}(t, x)\right), \quad i \in S, \\
u_{i}(t, x) & =\alpha_{i}(t, x), \quad(t, x) \in D_{0} \cup\left(\Sigma \backslash \Sigma_{c_{i}}\right), i \in S, \\
b_{i}(t, x) u_{i}(t, x) & -c_{i}(t, x) \frac{d u_{i}}{d l_{i}}(t, x)=\alpha_{i}(t, x), \quad(t, x) \in \Sigma_{c_{i}}, i \in S,
\end{aligned}
$$

where $u=\left\{u_{i}\right\}_{i \in S}$.

We say that a function $u=\left\{u_{i}\right\}_{i \in S}, u: \Delta \rightarrow X$, is a regular solution of system (1) if:

(i) $u \in C(\Delta, X)$,

(ii) for each $i \in S$ the derivatives $\partial_{t} u_{i}, \partial_{x} u_{i}, \partial_{x x} u_{i}$ exist and they are continuous in $D$,

(iii) $u$ satisfies system (1) for every $(t, x) \in D$.

If in addition, for every $i \in S$, the derivative $\frac{d u_{i}}{d l_{i}}(t, x)$ exists at each $(t, x) \in \Sigma_{c_{i}}$, then a regular solution is said to be $\Sigma_{c^{-}}$regular in $D$.

A $\Sigma_{c}$-regular solution $u=\left\{u_{i}\right\}_{i \in S}$ of (1) is called a parabolic solution of (1) in $D$ if for any two symmetric matrices $r, \widetilde{r} \in M_{n \times n}$ such that $r \leq \widetilde{r}$, the inequality

$$
f_{i}\left(t, x, u(t, x), u, \partial_{x} u_{i}(t, x), r\right) \leq f_{i}\left(t, x, u(t, x), u, \partial_{x} u_{i}(t, x), \widetilde{r}\right)
$$

is satisfied for $(t, x) \in D, i \in S$.

Differential systems with a deviated argument and differential-integral problems can be obtained from (1) by specializing the operator $f$. Note that various models of the functional dependence in partial equations are used in the literature. Detailed comparisons between different models are presented in [4]. 
The classical theory of parabolic differential inequalities has been described in the monographs [7]-[9], [16].

As is well known, they found applications in differential problems. The basic examples of such applications are: estimates of solutions of partial equations, estimates of the domain of existence of solutions, criteria of uniqueness and error estimates for approximate solutions. Moreover, discrete versions of differential inequalities are frequently used to prove convergence of approximation methods. The numerical method of lines and difference methods are classical examples.

Recently numerous papers have been published concerning parabolic functional-differential problems. Existence results can be found in [1]-[3]. They are based on the method of successive approximations introduced by T. Ważewski for systems without functional dependence [17]. The Chaplygin method is also used in existence theorems for parabolic functionaldifferential problems.

Functional-differential inequalities of parabolic type and uniqueness results for initial boundary value problems were first treated in [10]-[13]. Those papers deal with finite systems of weakly coupled functional-differential equations. This means that every equation contains the vector of unknown functions and the derivatives of only one function.

Infinite systems of parabolic functional-differential inequalities were considered in [15]. Monotonicity conditions and Lipschitz estimates are the main assumptions on the right hand sides of the system in that paper. Uniqueness results for infinite systems of parabolic functional-differential equations with initial boundary conditions can be found in [14]. The Lipschitz condition with respect to the functional variable is assumed in [14] and bounded solutions are considered. It is important in [14], [15] that the Lipschitz constant is common for all functions in the system of equations or inequalities. Similar problems for infinite systems and their solutions defined on unbounded domains and belonging to the function class $E_{2}^{1, \infty}$ were examined in [5], [6].

The aim of this paper is to study general comparison theorems for parabolic functional-differential infinite systems. The first part deals with extremal solutions of infinite systems of ordinary functional-differential equations. The second part contains comparison theorems for parabolic problems, uniqueness criteria for solutions of problem (1)-(3) and a result on continuous dependence of solutions on given functions.

It is essential to our considerations that the right hand sides of the equations satisfy nonlinear estimates of the Perron type with respect to the unknown functions. We deal with infinite systems of functional-differential equations as comparison problems for (1).

The paper generalizes results of [10], [11], [14] and [15]. 
2. Extremal solutions of ordinary functional-differential systems. Put $X_{+}=\left\{p=\left\{p_{k}\right\}_{k \in S}: p_{k} \geq 0, k \in S\right\}$. Let $C\left(\left[-r_{0}, a\right), X_{+}\right)$denote the space of all functions $w=\left\{w_{i}\right\}_{i \in S}$ such that $w_{i} \in C\left(\left[-r_{0}, a\right), \mathbb{R}_{+}\right)$, $i \in S$. Put $\widetilde{\Gamma}=(0, a) \times X_{+} \times C\left(\left[-r_{0}, a\right), X_{+}\right)$.

Assumption $\mathrm{H}[\sigma]$. Suppose that $\sigma=\left\{\sigma_{i}\right\}_{i \in S}, \sigma_{i} \in C\left(\widetilde{\Gamma}, \mathbb{R}_{+}\right)$, and

1) $\sigma$ satisfies the Volterra condition, i.e. for each $(t, p) \in(0, a) \times X_{+}$ and $w, \bar{w} \in C\left(\left[-r_{0}, a\right), X_{+}\right)$such that $w(\tau)=\bar{w}(\tau)$ for $\tau \in\left[-r_{0}, t\right]$ we have $\sigma(t, p, w)=\sigma(t, p, \bar{w})$

2) the following monotonicity condition holds: if $i \in S,(t, p, w),(t, \bar{p}, \bar{w})$ $\in \widetilde{\Gamma}, p=\left\{p_{i}\right\}_{i \in S}, \bar{p}=\left\{\bar{p}_{i}\right\}_{i \in S}$ and $p \leq \bar{p}, p_{i}=\bar{p}_{i}$, and $w(t) \leq \bar{w}(t)$ on $\left[-r_{0}, a\right)$ then $\sigma_{i}(t, p, w) \leq \sigma_{i}(t, \bar{p}, \bar{w})$,

3) there is $L \in X_{+}$such that

$$
\sigma(t, p, w) \leq L \quad \text { on } \widetilde{\Gamma} .
$$

Lemma 1. Suppose Assumption $\mathrm{H}[\sigma]$ is satisfied and $\eta \in C\left(\left[-r_{0}, 0\right], X_{+}\right)$. Then on $\left[-r_{0}, a\right)$ there exists the maximum solution $\omega(\cdot, \eta)=\left\{\omega_{i}(\cdot, \eta)\right\}_{i \in S}$ of the problem

$$
\begin{aligned}
w^{\prime}(t) & =\sigma(t, w(t), w), \\
w(t) & =\eta(t), \quad t \in\left[-r_{0}, 0\right] .
\end{aligned}
$$

Moreover, if $\varphi \in C\left(\left[-r_{0}, a\right), X_{+}\right)$satisfies the functional-differential inequality

$$
D_{-} \varphi(t) \leq \sigma(t, \varphi(t), \varphi)
$$

and the initial estimate $\varphi(t) \leq \eta(t), t \in\left[-r_{0}, 0\right]$, then

$$
\varphi(t) \leq \omega(t, \eta) \quad \text { for } t \in(0, a) .
$$

Proof. Let $\psi=\left\{\psi_{i}\right\}_{i \in S} \in C\left(\left[-r_{0}, a\right), X_{+}\right)$and take $k \in S, \xi \in$ $C\left(\left[-r_{0}, a\right), \mathbb{R}_{+}\right)$. Put

$$
P[k, \psi, \xi]=\left\{P_{i}[k, \psi, \xi]\right\}_{i \in S}, \quad P_{i}[k, \psi, \xi]= \begin{cases}\psi_{i} & \text { for } i \neq k \\ \xi & \text { for } i=k\end{cases}
$$

It follows $([8])$ that there exists the right hand maximum solution $W_{k}[\psi]$ of the Cauchy problem

$$
\begin{aligned}
\xi^{\prime}(t) & =\sigma_{k}(t, P[k, \psi, \xi](t), P[k, \psi, \xi]), \\
\xi(t) & =\eta_{k}(t), \quad t \in\left[-r_{0}, 0\right],
\end{aligned}
$$

and the solution is defined on $\left[-r_{0}, a\right)$. Put $W[\psi]=\left\{W_{k}[\psi]\right\}_{k \in S}$. It follows from the monotonicity condition for $\sigma$ that for $\psi, \widetilde{\psi} \in C\left(\left[-r_{0}, a\right), X_{+}\right)$such that $\psi(t) \leq \widetilde{\psi}(t)$ on $\left[-r_{0}, a\right)$ we have

$$
W[\psi](t) \leq W[\widetilde{\psi}](t) \quad \text { on }(0, a) .
$$


Denote by $\Omega$ the class of all $\psi \in C\left(\left[-r_{0}, a\right), X_{+}\right)$satisfying the differential inequality

$$
D_{-} \psi(t) \leq \sigma(t, \psi(t), \psi)
$$

and the initial estimate $\psi(t) \leq \eta(t), t \in\left[-r_{0}, 0\right]$.

The function

$$
\widetilde{\omega}_{k}(t)=\sup \left\{W_{k}[\psi](t): \psi \in \Omega\right\}, \quad t \in\left[-r_{0}, a\right), k \in S,
$$

exists and is continuous on $\left[-r_{0}, a\right)$. Moreover $\widetilde{\omega}_{k}(t)=\eta_{k}(t)$ for $t \in\left[-r_{0}, 0\right]$, $k \in S$. Thus $\widetilde{\omega}=\left\{\omega_{k}\right\}_{k \in S} \in C\left(\left[-r_{0}, a\right), X_{+}\right)$and $W[\psi](t) \leq W[\widetilde{\omega}](t)$ for $\psi \in \Omega, t \in(0, a)$. Therefore

$$
\widetilde{\omega}(t) \leq W[\widetilde{\omega}](t), \quad t \in(0, a) .
$$

On the other hand, we have

$$
\begin{aligned}
\frac{d}{d t} W_{k}[\widetilde{\omega}](t) & =\sigma_{k}\left(t, P\left[k, \widetilde{\omega}, W_{k}[\widetilde{\omega}]\right](t), P\left[k, \widetilde{\omega}, W_{k}[\widetilde{\omega}]\right]\right) \\
& \leq \sigma_{k}\left(t, P\left[k, W[\widetilde{\omega}], W_{k}[\widetilde{\omega}]\right](t), P\left[k,[\omega], W_{k}[\widetilde{\omega}]\right]\right)
\end{aligned}
$$

and consequently $W[\widetilde{\omega}] \in \Omega$. This gives

$$
\widetilde{\omega}(t) \geq W[\widetilde{\omega}](t), \quad t \in(0, a) .
$$

Inequalities (8) and (9) imply that $\widetilde{\omega}=W[\widetilde{\omega}]$. Thus $\widetilde{\omega}$ is the right hand maximum solution of $(4),(5)$.

It follows from (6) that $\varphi \in \Omega$ and this completes the proof.

Lemma 2. Suppose Assumption $\mathrm{H}[\sigma]$ is satisfied and $\eta \in C\left(\left[-r_{0}, 0\right], X_{+}\right)$. Let $\varphi=\left\{\varphi_{i}\right\}_{i \in S} \in C\left(\left[-r_{0}, a\right), X_{+}\right)$and let $\omega(\cdot, \eta)$ be the right hand maximum solution of (4), (5). Put

$$
J_{k}=\left\{\tau \in(0, a): \varphi_{k}(\tau)>\omega_{k}(\tau, \eta)\right\}, \quad k \in S .
$$

If for each $k \in S$,

$$
D_{-} \varphi_{k}(t) \leq \sigma_{k}(t, \varphi(t), \varphi)
$$

for $t \in J_{k}$, and $\varphi(t) \leq \eta(t)$ for $t \in\left[-r_{0}, 0\right]$ then

$$
\varphi(t) \leq \omega(t, \eta) \quad \text { for } t \in(0, a) .
$$

Proof. Let $k \in S$ and let $\gamma_{k}\left(\cdot, \eta_{k}\right)$ be the right hand maximum solution of the problem

$$
\begin{aligned}
\xi^{\prime}(t) & =\sigma_{k}(t, P[k, \varphi, \xi](t), P[k, \varphi, \xi]), \\
\xi(t) & =\eta_{k}(t), \quad t \in\left[-r_{0}, 0\right],
\end{aligned}
$$

where $P[k, \varphi, \xi]$ is given by (7) with $\psi=\varphi$.

Fix $k \in S$. For $t \in J_{k}$ we have

$$
D_{-} \varphi_{k}(t) \leq \sigma_{k}\left(t, P\left[k, \varphi, \varphi_{k}\right](t), P\left[k, \varphi, \varphi_{k}\right]\right)
$$


and $\varphi_{k}(t) \leq \eta_{k}(t), t \in\left[-r_{0}, 0\right]$. Thus

$$
\varphi_{k}(t) \leq \gamma_{k}\left(t, \eta_{k}\right) \quad \text { for } t \in(0, a)
$$

Put $\gamma(\cdot, \eta)=\left\{\gamma_{k}\left(\cdot, \eta_{k}\right)\right\}_{k \in S}$. We have

$$
\begin{aligned}
\frac{d}{d t} \gamma_{k}\left(t, \eta_{k}\right) & =\sigma_{k}\left(t, P\left[k, \varphi, \gamma_{k}\left(\cdot, \eta_{k}\right)\right](t), P\left[k, \varphi, \gamma_{k}\left(\cdot, \eta_{k}\right)\right]\right) \\
& \leq \sigma_{k}(t, \gamma(t, \eta), \gamma(\cdot, \eta)), \quad t \in(0, a), k \in S
\end{aligned}
$$

It follows from Lemma 1 that

$$
\gamma_{k}\left(t, \eta_{k}\right) \leq \omega_{k}(t, \eta), \quad t \in(0, a), k \in S,
$$

which together with (10) gives the assertion of Lemma 2.

3. Comparison theorems. We will estimate functions of several variables by means of functions of one variable. Therefore we will need the operator $V: C(\Delta, X) \rightarrow C\left(\left[-r_{0}, a\right), X_{+}\right)$defined in the following way: for $z=\left\{z_{i}\right\}_{i \in S} \in C(\Delta, X)$ and $t \in\left[-r_{0}, a\right)$ let $V z=\left\{V_{i} z\right\}_{i \in S}$ where

$$
\left(V_{i} z\right)(t)=\max \left\{\left|z_{i}(t, x)\right|: x \in \bar{G}\right\}, \quad i \in S .
$$

For $p=\left\{p_{i}\right\}_{i \in S} \in X$ write $|p|=\left\{\left|p_{i}\right|\right\}_{i \in S}$.

TheOREm 1. Suppose that Assumption $\mathrm{H}[\sigma]$ is satisfied and the function $f=\left\{f_{i}\right\}_{i \in S}, f_{i}: \Gamma \rightarrow \mathbb{R}$, satisfies the estimates

$$
f_{i}(t, x, p, z, 0,0) \operatorname{sign} p_{i} \leq \sigma_{i}(t,|p|, V z), \quad i \in S,
$$

where $(t, x, p, z) \in D \times X \times C(\Delta, X)$. Suppose that $u=\left\{u_{i}\right\}_{i \in S}$ is a $\Sigma_{c^{-}}$ regular parabolic solution of (1) in $D$ satisfying the initial inequalities

$$
\left|u_{i}(t, x)\right| \leq \eta_{i}(t), \quad(t, x) \in D_{0}, i \in S,
$$

and boundary inequalities

$$
\begin{gathered}
\left|u_{i}(t, x)\right| \leq \omega_{i}(t, \eta), \quad(t, x) \in \Sigma \backslash \Sigma_{c_{i}}, i \in S, \\
\left|b_{i}(t, x) u_{i}(t, x)-c_{i}(t, x) \frac{d u_{i}}{d l_{i}}(t, x)\right| \leq B_{i} \omega_{i}(t, \eta), \quad(t, x) \in \Sigma_{c_{i}}, i \in S,
\end{gathered}
$$

where $\eta=\left\{\eta_{i}\right\}_{i \in S} \in C\left(\left[-r_{0}, 0\right], X\right)$ and $\omega(\cdot, \eta)=\left\{\omega_{i}(\cdot, \eta)\right\}_{i \in S}$ is the maximum solution of (4), (5). Under these assumptions we have

$$
|u(t, x)| \leq \omega(t, \eta) \quad \text { on } \Delta .
$$

Proof. Put $W_{i}(t)=\max \left\{\left|u_{i}(t, x)\right|: x \in \bar{G}\right\}$ for $i \in S, t \in\left[-r_{0}, a\right)$ and let $W=\left\{W_{i}\right\}_{i \in S}$. We will prove that for fixed $i \in S$,

$$
D_{-} W_{i}(t) \leq \sigma_{i}(t, W(t), W)
$$

where $t \in E_{i}=\left\{t^{\prime} \in(0, a): W_{i}\left(t^{\prime}\right)>\omega_{i}\left(t^{\prime}, \eta\right)\right\}$. Fix $\tilde{t} \in E_{i}$. There is $x_{i} \in \bar{G}$ such that
(a) $W_{i}(\widetilde{t})=u_{i}\left(\widetilde{t}, x_{i}\right) \quad$ or
(b) $W_{i}(\widetilde{t})=-u_{i}\left(\widetilde{t}, x_{i}\right)$. 
Consider the case (a). We conclude that $\left(\tilde{t}, x_{i}\right)$ is an interior point of $D$ and thus $\partial_{x} u_{i}\left(\widetilde{t}, x_{i}\right)=0, \partial_{x x} u_{i}\left(\widetilde{t}, x_{i}\right) \leq 0$. We obtain

$$
\begin{aligned}
D_{-} W_{i}(\widetilde{t}) & \leq \partial_{t} u_{i}\left(\widetilde{t}, x_{i}\right)=f_{i}\left(\widetilde{t}, x_{i}, u\left(\widetilde{t}, x_{i}\right), u, \partial_{x} u_{i}\left(\widetilde{t}, x_{i}\right), \partial_{x x} u_{i}\left(\widetilde{t}, x_{i}\right)\right) \\
& \leq f_{i}\left(\widetilde{t}, x_{i}, u\left(\widetilde{t}, x_{i}\right), u, 0,0\right) \leq \sigma_{i}\left(\widetilde{t},\left|u\left(\widetilde{t}, x_{i}\right)\right|, V u\right) \leq \sigma_{i}(\widetilde{t}, W(\widetilde{t}), W) .
\end{aligned}
$$

If we consider the case (b) then $\partial_{x} u_{i}\left(\tilde{t}, x_{i}\right)=0$ and $-\partial_{x x} u_{i}\left(\widetilde{t}, x_{i}\right) \leq 0$. Since $D_{-} W_{i}(\widetilde{t}) \leq-\partial_{t} u_{i}\left(\widetilde{t}, x_{i}\right)$ and $\operatorname{sign} u_{i}\left(\widetilde{t}, x_{i}\right)=-1$ we have

$$
\begin{aligned}
D_{-} W_{i}(\widetilde{t}) & \leq f_{i}\left(\tilde{t}, x_{i}, u\left(\tilde{t}, x_{i}\right), u, \partial_{x} u_{i}\left(\tilde{t}, x_{i}\right), \partial_{x x} u_{i}\left(\tilde{t}, x_{i}\right)\right) \operatorname{sign} u_{i}\left(\tilde{t}, x_{i}\right) \\
& \leq f_{i}\left(\tilde{t}, x_{i}, u\left(\tilde{t}, x_{i}\right), u, 0,0\right) \operatorname{sign} u_{i}\left(\widetilde{t}, x_{i}\right) \leq \sigma_{i}(\tilde{t}, W(\widetilde{t}), W) .
\end{aligned}
$$

The assertion follows from Lemma 2.

THEOREM 2. Suppose that the function $\sigma=\left\{\sigma_{i}\right\}_{i \in S}$ satisfies Assumption $\mathrm{H}[\sigma]$ and the functions $f=\left\{f_{i}\right\}_{i \in S}, f_{i}: \Gamma \rightarrow \mathbb{R}, g=\left\{g_{i}\right\}_{i \in S}$, $g_{i}: \Gamma \rightarrow \mathbb{R}$ satisfy the estimates

$$
\begin{aligned}
\left(f_{i}(t, x, p, z, q, r)-g_{i}(t, x, \bar{p}, \bar{z}, q, r)\right) & \operatorname{sign}\left(p_{i}-\bar{p}_{i}\right) \\
\leq & \sigma_{i}(t,|p-\bar{p}|, V(z-\bar{z})), \quad i \in S,
\end{aligned}
$$

for $(t, x, p, z, q, r),(t, x, \bar{p}, \bar{z}, q, r) \in \Gamma$. Suppose that $u=\left\{u_{i}\right\}_{i \in S}$ is a $\Sigma_{c^{-}}$ regular parabolic solution of (1) in $D$ and $v=\left\{v_{i}\right\}_{i \in S}$ is a $\Sigma_{c}$-regular solution of the system

$$
\partial_{t} v_{i}(t, x)=g_{i}\left(t, x, v(t, x), v, \partial_{x} v_{i}(t, x), \partial_{x x} v_{i}(t, x)\right), \quad i \in S .
$$

Let the initial inequalities

$$
\left|u_{i}(t, x)-v_{i}(t, x)\right| \leq \eta_{i}(t, x), \quad(t, x) \in D_{0}, i \in S,
$$

and boundary inequalities

$$
\begin{gathered}
\left|u_{i}(t, x)-v_{i}(t, x)\right| \leq \omega_{i}(t, \eta), \quad(t, x) \in \Sigma \backslash \Sigma_{c_{i}}, i \in S, \\
\left|b_{i}(t, x)\left(u_{i}(t, x)-v_{i}(t, x)\right)-c_{i}(t, x) \frac{d\left(u_{i}-v_{i}\right)}{d l_{i}}(t, x)\right| \leq B_{i} \omega_{i}(t, \eta), \\
(t, x) \in \Sigma_{c_{i}}, i \in S,
\end{gathered}
$$

where $\eta=\left\{\eta_{i}\right\}_{i \in S} \in C\left(\left[-r_{0}, 0\right], X_{+}\right)$, hold true. Under these assumptions we have

$$
\left|u_{i}(t, x)-v_{i}(t, x)\right| \leq \omega_{i}(t, \eta), \quad(t, x) \in D, i \in S
$$

Proof. Put $W_{i}(t)=\max \left\{\left|u_{i}(t, x)-v_{i}(t, x)\right|: x \in \bar{G}\right\}$ for $i \in S, t \in$ $\left[-r_{0}, a\right)$. Fix $i \in S$. Let $W_{i}(\widetilde{t})>\omega_{i}(\widetilde{t}, \eta)$. There is $x_{i} \in \bar{G}$ such that
(a) $W_{i}(\widetilde{t})=u_{i}\left(\widetilde{t}, x_{i}\right)-v_{i}\left(\tilde{t}, x_{i}\right)$ or
(b) $W_{i}(\widetilde{t})=-\left(u_{i}\left(\tilde{t}, x_{i}\right)-v_{i}\left(\tilde{t}, x_{i}\right)\right)$. 
In case (a) we obtain $\partial_{x} u_{i}\left(\widetilde{t}, x_{i}\right)=\partial_{x} v_{i}\left(\widetilde{t}, x_{i}\right), \partial_{x x} u_{i}\left(\widetilde{t}, x_{i}\right) \leq \partial_{x x} v_{i}\left(\widetilde{t}, x_{i}\right)$ and $D_{-} W_{i}(\tilde{t}) \leq \partial_{t} u_{i}\left(\tilde{t}, x_{i}\right)-\partial_{t} v_{i}\left(\tilde{t}, x_{i}\right)$

$$
\begin{aligned}
\leq & f_{i}\left(\widetilde{t}, x_{i}, u_{i}\left(\widetilde{t}, x_{i}\right), u, \partial_{x} u_{i}\left(\widetilde{t}, x_{i}\right), \partial_{x x} u_{i}\left(\widetilde{t}, x_{i}\right)\right) \\
& -f_{i}\left(\widetilde{t}, x_{i}, u_{i}\left(\widetilde{t}, x_{i}\right), u, \partial_{x} u_{i}\left(\widetilde{t}, x_{i}\right), \partial_{x x} v_{i}\left(\tilde{t}, x_{i}\right)\right) \\
& +f_{i}\left(\widetilde{t}, x_{i}, u_{i}\left(\widetilde{t}, x_{i}\right), u, \partial_{x} u_{i}\left(\widetilde{t}, x_{i}\right), \partial_{x x} v_{i}\left(\widetilde{t}, x_{i}\right)\right) \\
& -g_{i}\left(\widetilde{t}, x_{i}, v_{i}\left(\widetilde{t}, x_{i}\right), v, \partial_{x} u_{i}\left(\widetilde{t}, x_{i}\right), \partial_{x x} v_{i}\left(\widetilde{t}, x_{i}\right)\right) \leq \sigma_{i}(\widetilde{t}, W(\widetilde{t}), W),
\end{aligned}
$$

and analogously in case (b). The above inequalities and Lemma 2 complete the proof.

An immediate consequence of Theorem 2 is the following uniqueness theorem.

THEOREM 3. Suppose that the function $\sigma=\left\{\sigma_{i}\right\}_{i \in S}$ satisfies Assumption $\mathrm{H}[\sigma]$ and $w(t)=0, t \in\left[-r_{0}, a\right)$, is a unique solution of (4) such that $w(t)=0$ for $t \in\left[-r_{0}, 0\right]$, i.e. $\omega(t, 0)=0$. Let the function $f=\left\{f_{i}\right\}_{i \in S}$, $f_{i}: \Gamma \rightarrow \mathbb{R}$, satisfy the estimates

$$
\begin{aligned}
\left(f_{i}(t, x, p, z, q, r)-f_{i}(t, x, \bar{p}, \bar{z}, q, r)\right) & \operatorname{sign}\left(p_{i}-\bar{p}_{i}\right) \\
\leq & \sigma_{i}(t,|p-\bar{p}|, V(z-\bar{z})), \quad i \in S,
\end{aligned}
$$

for $(t, x, p, z, q, r),(t, x, \bar{p}, \bar{z}, q, r) \in \Gamma$. Then the problem (1)-(3) admits at most one $\Sigma_{c}$-regular parabolic solution in $D$.

The next theorem concerns continuous dependence of solutions.

THEOREM 4. Suppose that all assumptions of Theorem 3 hold true and $u=\left\{u_{i}\right\}_{i \in S}$ is a $\Sigma_{c}$-regular parabolic solution of (1)-(3) in D. For $\varepsilon>0$ and $f^{\varepsilon}=\left\{f_{i}^{\varepsilon}\right\}_{i \in S}, f_{i}^{\varepsilon}: \Gamma \rightarrow \mathbb{R}, \alpha^{\varepsilon}=\left\{\alpha_{i}^{\varepsilon}\right\}_{i \in S}, \alpha_{i}^{\varepsilon}: D_{0} \cup \Sigma \rightarrow \mathbb{R}$, let $v^{\varepsilon}=\left\{v_{i}^{\varepsilon}\right\}_{i \in S}$ be a $\Sigma_{c}$-regular solution in $D$ of the problem

$$
\begin{gathered}
\partial_{t} v_{i}(t, x)=f_{i}^{\varepsilon}\left(t, x, v(t, x), v, \partial_{x} v_{i}(t, x), \partial_{x x} v_{i}(t, x)\right), \quad i \in S, \\
v_{i}(t, x)=\alpha_{i}^{\varepsilon}(t, x) \quad \text { on } D_{0} \cup\left(\Sigma \backslash \Sigma_{c_{i}}\right), i \in S, \\
b_{i}(t, x) v_{i}^{\varepsilon}(t, x)-c_{i}(t, x) \frac{d v_{i}^{\varepsilon}}{d l_{i}}(t, x)=\alpha_{i}^{\varepsilon}(t, x), \quad(t, x) \in \Sigma_{c_{i}}, \quad i \in S .
\end{gathered}
$$

Suppose that

$$
\begin{aligned}
& \left|f_{i}(t, x, p, z, q, r)-f_{i}^{\varepsilon}(t, x, p, z, q, r)\right| \leq \varepsilon \quad \text { on } \Gamma, \\
& \left|\alpha(t, x)-\alpha^{\varepsilon}(t, x)\right| \leq \varepsilon \quad \text { on } D_{0} \cup \Sigma \text {. }
\end{aligned}
$$

Denote by $\omega^{\varepsilon}=\left\{\omega_{i}^{\varepsilon}\right\}_{i \in S}$ the right hand maximum solution of the infinite system

$$
w_{i}^{\prime}(t)=\sigma_{i}(t, w(t), w)+\varepsilon, \quad i \in S,
$$

with the initial conditions

$$
w_{i}(t)=\max \left\{\varepsilon, \varepsilon / B_{i}\right\} \quad \text { on }\left[-r_{0}, 0\right], i \in S .
$$


Under these assumptions

$$
\left|v_{i}^{\varepsilon}(t, x)-u_{i}(t, x)\right| \leq \omega_{i}^{\varepsilon}(t) \quad \text { on } D \text { for } i \in S .
$$

Proof. Fix $i \in S$. We have

$\left(f_{i}(t, x, p, z, q, r)-f_{i}^{\varepsilon}(t, x, \bar{p}, \bar{z}, q, r)\right) \operatorname{sign}\left(p_{i}-\bar{p}_{i}\right) \leq \sigma_{i}(t,|p-\bar{p}|, V(z-\bar{z}))+\varepsilon$ for $(t, x, p, z, q, r),(t, x, \bar{p}, \bar{z}, q, r) \in \Gamma$. Since $\omega^{\varepsilon}$ is nondecreasing and $\omega_{i}^{\varepsilon}(0)=$ $\max \left\{\varepsilon, \varepsilon / B_{i}\right\}$ we get $\varepsilon \leq B_{i} \omega_{i}^{\varepsilon}(t), t \in(0, a), i \in S$. Theorem 2 now yields the assertion.

REMARK 1. If all the assumptions of Theorem 4 are satisfied then for each $i \in S$ we have

$$
\lim _{\varepsilon \rightarrow 0} \omega_{i}^{\varepsilon}(t)=0 \quad \text { uniformly on }\left[-r_{0}, a\right) .
$$

Indeed, for each $i \in S$, the functions $\omega_{i}^{\varepsilon}:\left[-r_{0}, a\right) \rightarrow \mathbb{R}, \varepsilon>0$, satisfy the assumptions of the Ascoli-Arzelà theorem and they are nondecreasing with respect to $\varepsilon$. Hence the assertion follows.

\section{References}

[1] S. Brzychczy, Approximate iterative method and the existence of solutions of nonlinear parabolic differential-functional equations, Ann. Polon. Math. 42 (1983), 3743.

[2] - Existence of solutions for non-linear systems of differential-functional equations of parabolic type in an arbitrary domain, ibid. 47 (1987), 309-317.

[3] - Existence of solutions and monotone iterative method for infinite systems of parabolic differential-functional equations, ibid. 72 (1999), 15-24.

[4] Z. Kamont, Initial value problems for hyperbolic differential-functional systems, Boll. Un. Mat. Ital. B (7) 8 (1994), 965-984.

[5] B. Kraśnicka, On some properties of solutions to a mixed problem for an infinite system of parabolic differential-functional equations in an unbounded domain, Demonstratio Math. 15 (1982), 229-240.

[6] -, On some properties of solutions to the first Fourier problem for infinite system of parabolic differential-functional equations in an arbitrary domain, Univ. Iagell. Acta Math. 26 (1987), 67-74.

[7] G. S. Ladde, V. Lakshmikantham and A. S. Vatsala, Monotone Iterative Techniques for Nonlinear Differential Equations, Pitman, Boston, 1985.

[8] V. Lakshmikantham and S. Leela, Differential and Integral Inequalities, Academic Press, New York, 1969.

[9] J. Szarski, Differential Inequalities, Polish Sci. Publ., Warszawa, 1967.

[10] - Uniqueness of solutions of a mixed problem for parabolic differential-functional equations, Ann. Polon. Math. 28 (1973), 57-65.

[11] —, Strong maximum principle for non-linear parabolic differential-functional inequalities, ibid. 29 (1974), 207-214.

[12] - Strong maximum principle for non-linear parabolic differential-functional inequalities in arbitrary domains, ibid. 31 (1975), 197-203. 
[13] J. Szarski, Uniqueness of the solution to a mixed problem for parabolic functionaldifferential equations in arbitrary domains, Bull. Acad. Polon. Sci. Sér. Sci. Math. Astronom. Phys. 24 (1976), 841-849.

[14] -, Comparison theorem for infinite systems of parabolic differential-functional equations and strongly coupled infinite systems of parabolic equations, ibid. 27 (1979), 839-846.

[15] —, Infinite systems of parabolic differential-functional inequalities, ibid. 28 (1980), 477-481.

[16] W. Walter, Differential and Integral Inequalities, Springer, Berlin, 1970.

[17] T. Ważewski, Sur le problème de Cauchy relatif à un système d'équations aux dérivées partielles, Ann. Soc. Polon. Math. 15 (1936), 101-127.

Institute of Mathematics

University of Gdańsk

Wita Stwosza 57

80-952 Gdańsk, Poland

E-mail: dana@math.univ.gda.pl 